\title{
Computerized Kymograph for Muscle Contraction Measurement Using Ultrasonic Distance Sensor
}

\author{
Suhaeri $^{1}$, Vitri Tundjungsari ${ }^{2}$ \\ YARSI University \\ Faculty of Information Technology (FTI) \\ J1. Letjen Suprapto, Jakarta Pusat, Indonesia
}

\begin{abstract}
Kymograph is a device to record the magnitude of physiological variables, such as: muscle contraction. However, we observe some lacks of the conventional kymographs, such as: result's visualisation and accuracy. Hence, we propose a computerized-kymograph which can automatically measure, record, and display graphical data of muscle contraction on the computer by using ultrasonic distance sensor. We develop hardware and software systems to support computerized kymograph and then test our device with live frog. The result shows that the device works well by displaying better visualization than the conventional kymograph.
\end{abstract}

Keywords—kymograph; computerized; distance sensor

\section{INTRODUCTION}

Kymograph is an aid for recording the magnitude as well as time course of a wide variety of physiological variables. The fundamental principle of converting time into distance has remained unchanged from the kymograph through polygraphs and oscilloscopes. We combine the fundamental works of conventional kymograph and a computerized device. A computerized device is defined as a machine that performs calculations and processes automatically. Hence, we define computerized kymograph as an automatic device that performs kymograph functionality, by recording and displaying the result on the computer.

We observe that the conventional kymograph at our laboratory having some lacks of visual appearance and accuracy. For example, the conventional kymograph did not demonstrate the value of muscle's contraction. Therefore we propose the computerized kymograph to perform better than the conventional one.

This paper presents an automated system to: (i) generate and display graphical data by computer, (ii) enhance the conventional kymographs result visibility for more accurate result. Section 2 discusses some related works about kymograph and its application. Section 3 explains how the conventional kymograph works and why we need to improve its performance. Section 4 describes how computerized kymograph is developed and tested. In section 4, we also explain the steps of the algorithm. Section 5 discusses the conclusion of our proposed device.

\section{RELATED WORKS}

There are several works have been done related to kymograph for motion analysis and detection. Spatiotemporal method has been used in kymograph for motion analysis and detection, such as: using kymograph of video sequences to form a spatiotemporal image representation [1-4]. Kymographs have also been used to track image features over time; for example, the algorithm in [1,3] used a variable-rate particle filter to enhance the accuracy of the extracted edges corresponding to the tip of micro-tubules from a kymographlike image representation.

Mukherjee et al. [1] propose an automated method to profile the velocity patterns of small organelles (BDNF granules) being transported along a selected section of axon of a cultured neuron imaged by time-lapse fluorescence microscopy. The proposed method starts by generating a twodimensional spatiotemporal map (kymograph) of the granule traffic along an axon segment, instead of directly detecting the granules as in conventional tracking. Author in [3] evaluated axonal transport by cross- and auto-correlation of kymograph columns; while author in [2] generated kymographs by hand and analysed them using the Radon transform to detect peak velocities of particles.

Our paper proposes a computerized-based kymograph in order to study the characteristics of certain physiological events such as muscle contractions, by providing more accurate recording device than a conventional kymograph. We argue that our proposed device can provide more accurate result of various physiological changes because it can directly record and display the skeletal muscle contractions (muscles can be removed from anesthetized frogs) on the computer. These muscles can be attached to recording systems and measured by ultrasonic distance sensor, stimulated by electrical shocks of varying strength, duration, and frequency. Recordings obtained from such procedures can be used to study the basic characteristics of skeletal muscle contractions.

\section{RESEARCH BACKGROUND}

Our research starts from Indonesian's medical faculty for physiology experiment by observing live frog's muscle contraction using conventional kymograph as a device. However, we notice some weaknesses from conventional kymograph's usage in order to observe and record muscle contraction.

Before discussing how our proposed device can overcome conventional kymograph's weaknesses, we will discuss the principal works of conventional kymograph. 


\section{A. How Conventional Kymograph Works}

To observe the phenomenon of skeletal muscle contractions, muscles can be removed from anesthetized frogs. These muscles can be attached to kymograph's recording systems and stimulated by electrical shocks of varying strength, duration, and frequency. Recordings obtained from such procedures can be used to study the basic characteristics of skeletal muscle contractions. Figure 1 and 2 show the kymograph from upper and front view. The procedures of using conventional kymograph are described as follows:

- The Kymograph, which is used to study muscle physiology, consists of a drum, which rotates at a preset speed, and traces are produced on the paper by means of an ink writing pointer. Thus, the drum can be rotated rapidly if rapid physiological events are being recorded or rotated slowly for events that occur more slowly.

- A stylus that can mark on the paper is attached to a movable lever, and the lever, in turn, is connected to an isolated muscle. The origin of the muscle is fixed in position by a clamp, and its insertion is hooked to the muscle lever.

- To study muscle physiology, a simple experiment can be carried out by using the frog gastrocnemius muscle/sciatic nerve. The principles of muscle excitation, contraction and work performed in the frog are similar to all vertebrates including man. Using frog tissue has the practical advantage that it will function at room temperature without a blood supply; its oxygen requirements are met by diffusion from the air into the solution bathing the preparation.

- The muscle also is connected by wires to an electronic stimulator. The stimulator within the kymograph delivers small electric pulses to the muscle or nerve tissue via a pair of platinum electrodes.

- The stimulus can deliver single pulses or repeated stimulation up to a frequency of 100 per sec. An audible click is produced when a pulse is delivered. The stimulator may also be triggered using the trigger switch attached to the kymograph spindle.

\section{B. The Need for Computerized Kymograph}

Based on our experiment and observation, we notice that conventional kymograph has some weaknesses, such as: damping risk on muscle lever caused by friction between the lever and the kymograph's drum; therefore the result recorded is not accurate as it is supposed to be.

Hence, our proposed computerized-based kymograph could overcome the problem because the result recorded immediately afterward the sensor get the distance as muscle contraction result. We also argue that our device produces more visible graphical result and also easier to use than the conventional kymograph.
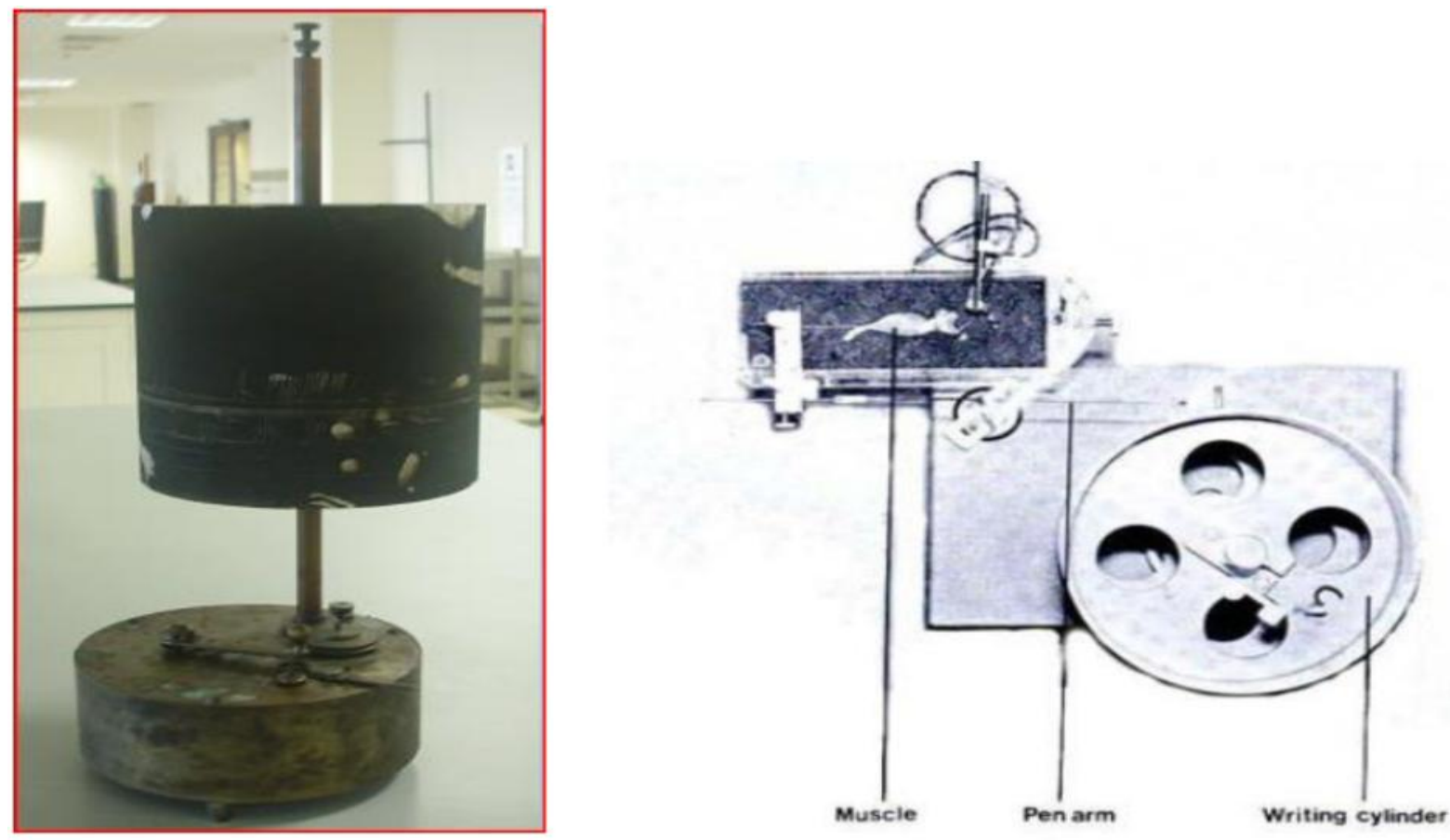

Fig. 1. Conventional Kymograph (front view)

Fig. 2. Conventional Kymograph (upper view) 


\section{CONSTRUCTING COMPUTERIZED KYMOGRAPH}

This section discusses how computerized-based kymograph is developed. The device consists of hardware and software systems, as discussed below.

\section{A. Hardware System}

The hardware system consists of two main parts, which are: (1) ultrasonic distance sensor and (2) microcontroller as an interface between the sensor and the computer. The sensor works in the following ways: A sonic pulse is emitted from the sensor; then when the pulse bounces off of an object, an echo is returned. The sensor is able to emit the pulse because of a transducer that converts between sonic, electrical and mechanical energies.

We use ultrasonic sensor because it provides an easy method of distance measurement and has easy interfacing. This sensor is perfect for any number of applications that require to perform measurements between moving or stationary objects [6]. Some application ideas that can use this sensor are: security systems, interactive animated exhibits, parking assistant systems, and robotic navigation. The key features in this ultrasonic sensor are [6]: (1) provides precise, non-contact distance measurements within a $2 \mathrm{~cm}$ to $3 \mathrm{~m}$ range; (2) ultrasonic measurements work in any lighting condition, making this a good choice to supplement infrared object detectors; (3) simple pulse in/pulse out communication requires just one I/O pin; (4) burst indicator LED shows measurement in progress; (5) 3-pin header makes it easy to connect to a development board, directly or with an extension cable, hence no soldering required.

We use the sensor to measure the distance of muscle contraction. The sensor measures the time required for the echo return, and returns this value to the microcontroller as a variable-width pulse via the same I/O pin. The sensor works as an input to the microcontroller by sending the data continuously and display the graphical image on the computer display (by setting timer initialization). Figure 3 shows the ultrasonic distance sensor, and figure 4 shows how the sensor works during the time.

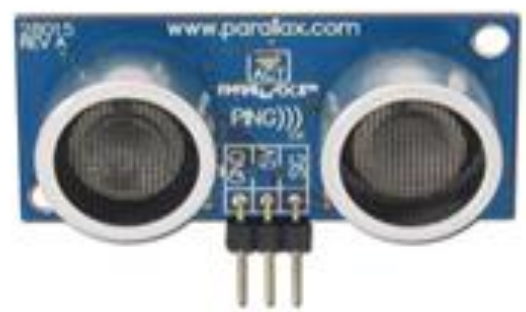

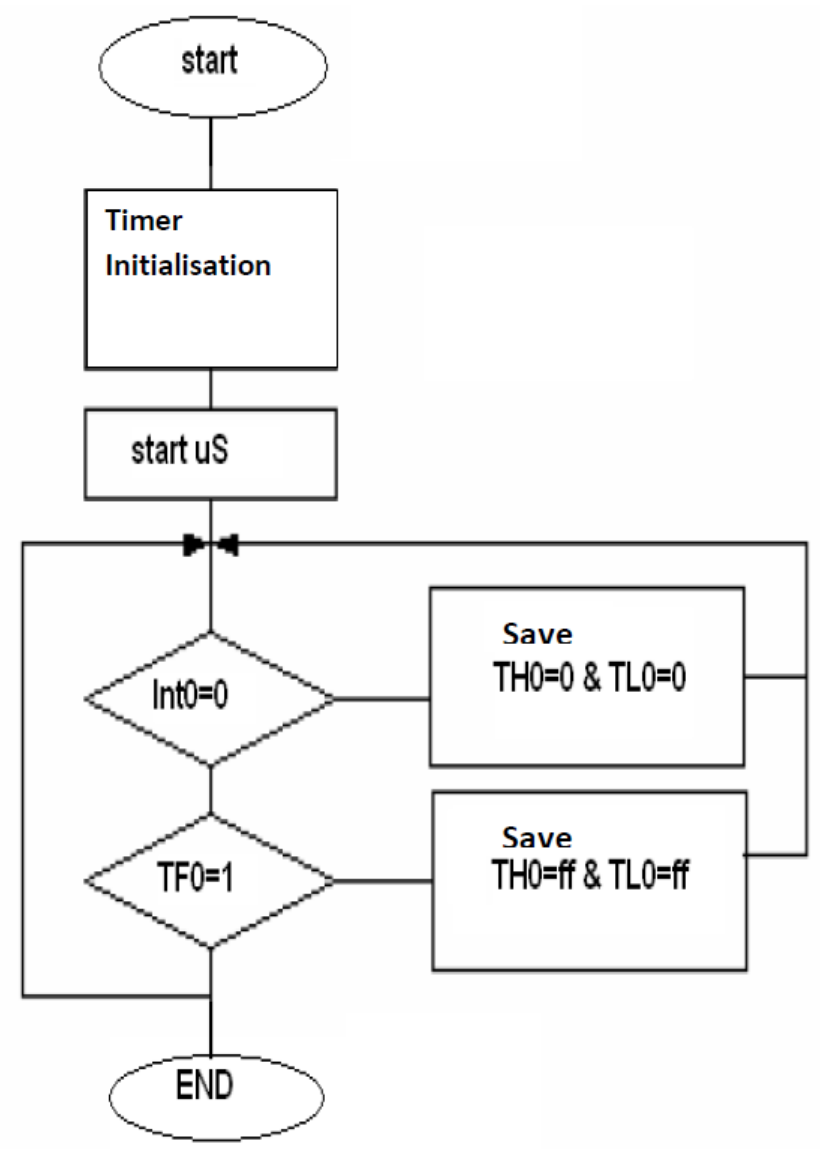

Fig. 4. Flowchart of Ultrasonic distance sensor

We also use microcontroller based on the AVR enhanced RISC architecture. By executing instructions in a single clock cycle, the ATmega8535 achieves throughputs approaching 1 MIPS per MHz allowing the system's designer to optimize power consumption versus processing speed [5]. Figure 5 shows the components of microcontroller.

Figure 6 demonstrates all stages required for the device as a complete system. The system starts whenever the frog's muscle is pinched (figure 10), the device will recognized a distance disparity using ultrasonic distance sensor. Having the distance data, the device will read and process it (figure 8). Those data processing and calculating are performed using Microkontroller ATMEGA 8535. Then, using serial port the data will be delivered and displayed to computer, as a data receiver and recorder (figure 8).

Fig. 3. Ultrasonic distance sensor [6] 


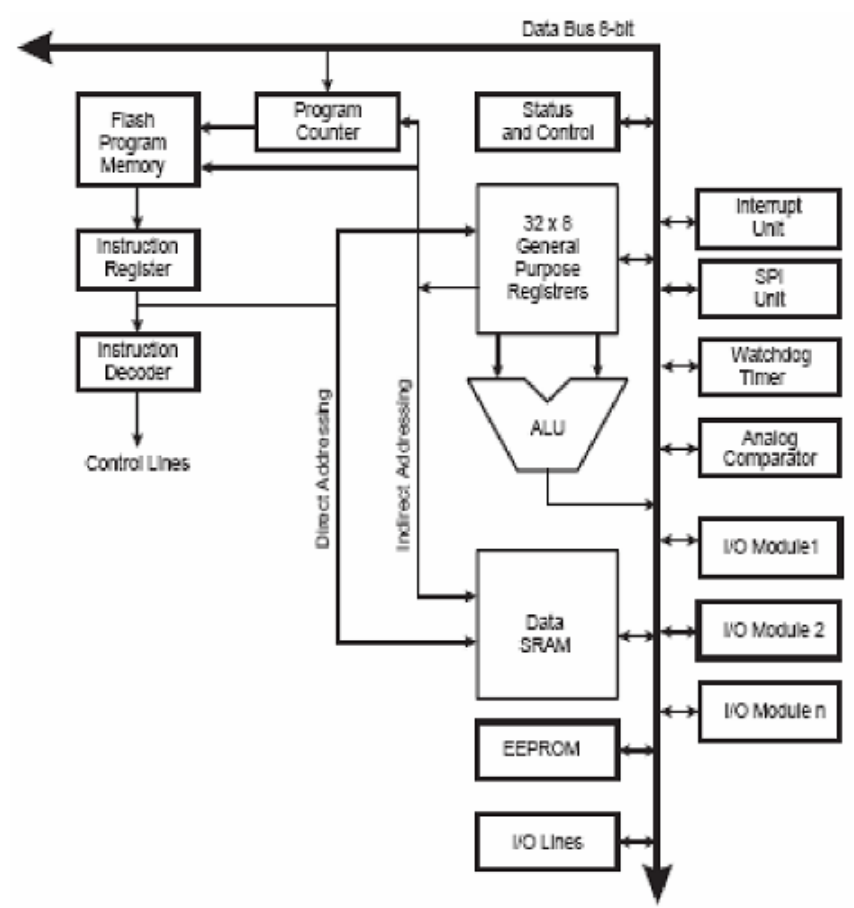

Fig. 5. Flowchart of Ultrasonic distance sensor [5]

\section{B. Software System}

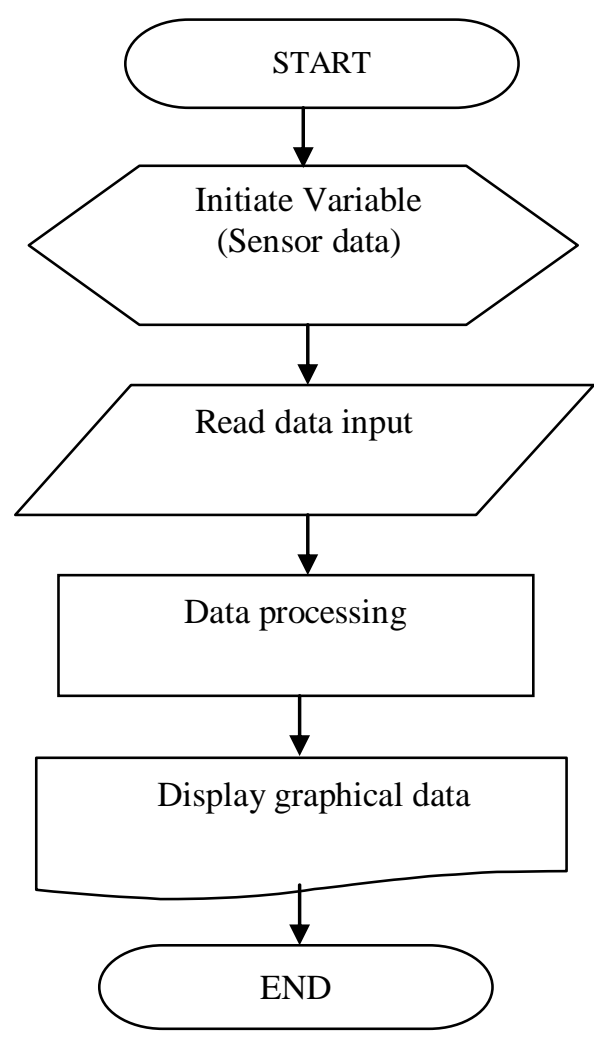

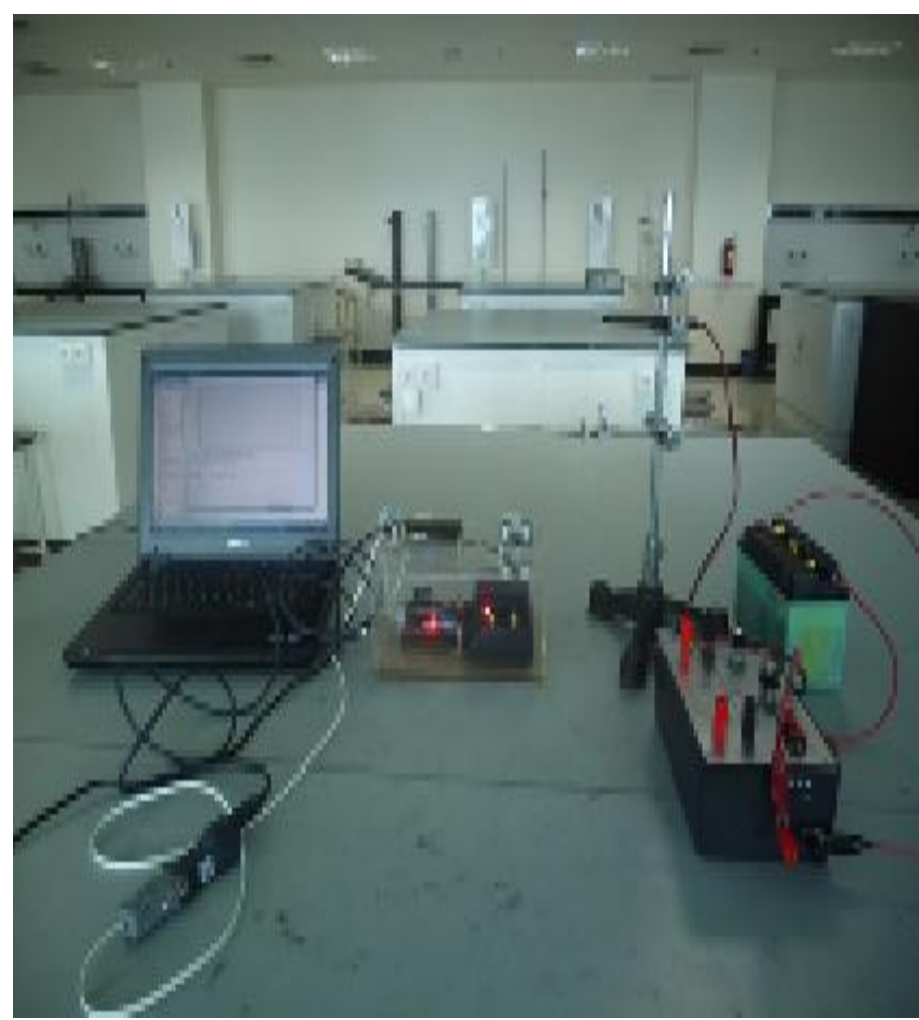

Fig. 7. Computerized kymograph system

Figure 7 shows how software system in the device is connected to hardware system. Ultrasonic sensor is employed to measuring the distance caused by muscle contraction over a period of time.

The result is then being processed and calculated by means of microcontroller. The microcontroller also used as an interface device to display the graphical image on the computer's display. Figure 9 demonstrates how the sensor and microcontroller connected tocomputerized-kymograph.

\section{Device Testing}

In this section, we will discuss the device's result compare to the conventional kymograph. Figure 10 demonstrate a live frog's muscle to be tested by our computerized kymograph device.

These frog's muscles can be attached to kymograph's recording systems and stimulated by electrical shocks of varying strength, duration, and frequency. Recordings obtained from the sensor and processed by microcontroller. The result is then displayed on the computer.

Figure 11 displays the result on conventional kymograph; while figure 12 displays the result on computerized kymograph. We can see from both results that computerized kymograph (figure 11) shows more visible result than conventional kymograph, by providing muscle contraction value (figure 12).

Fig. 6. Software system in Computerized kymograph algorithm 


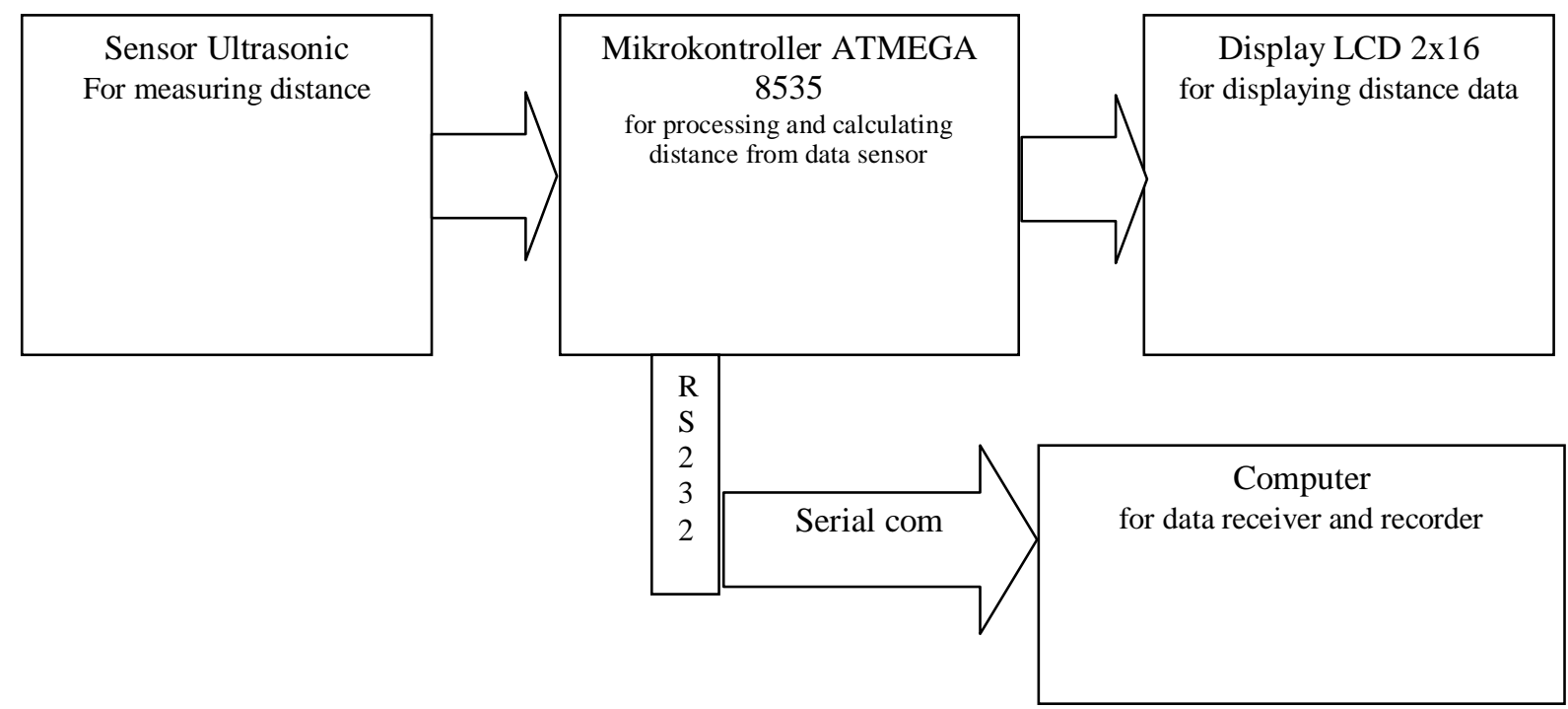

Fig. 8. Hardware system in Computerized kymograph

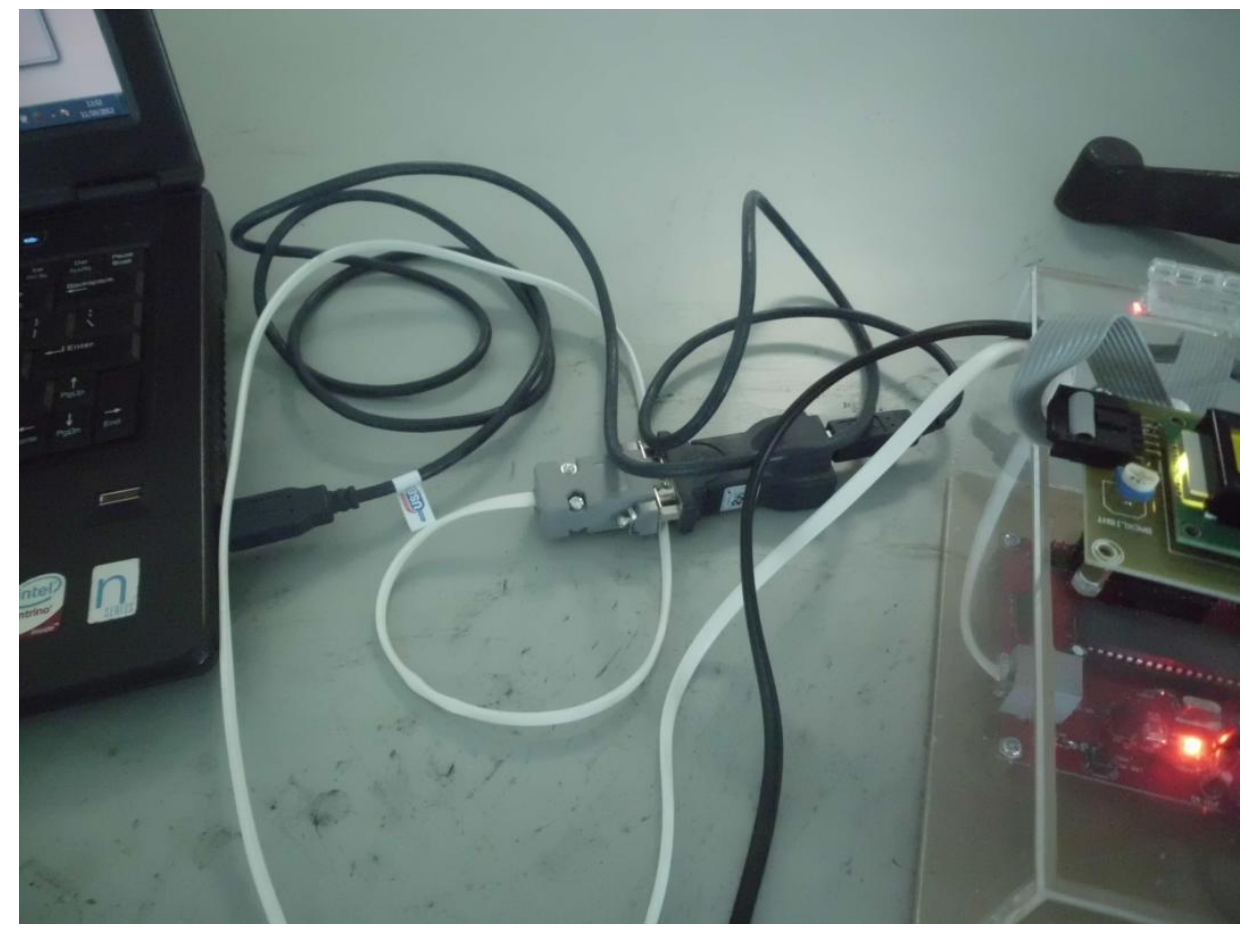

Fig. 9. Sensor and microcontroller used in computerized-kymograph

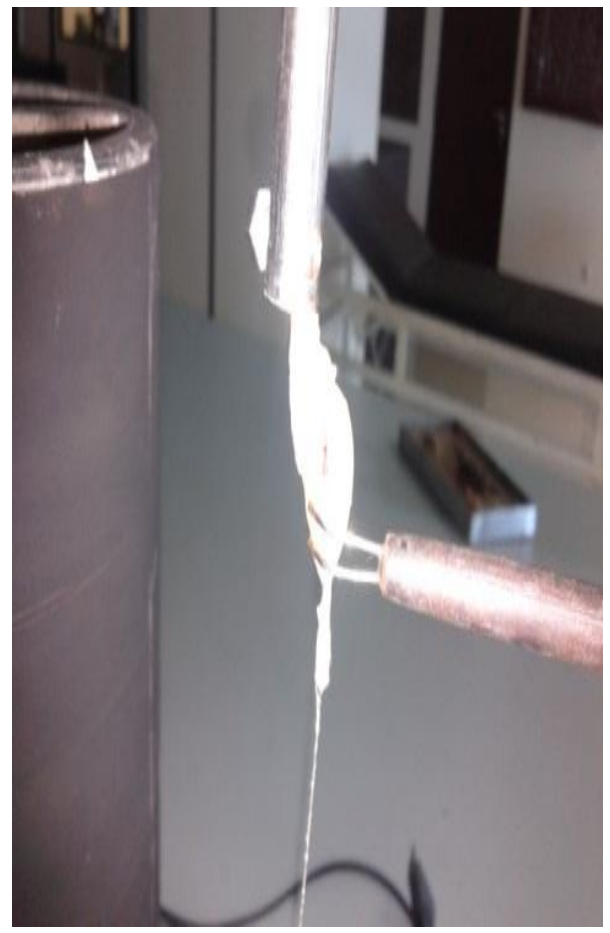

Fig. 10. Frog's muscle used in experiment 


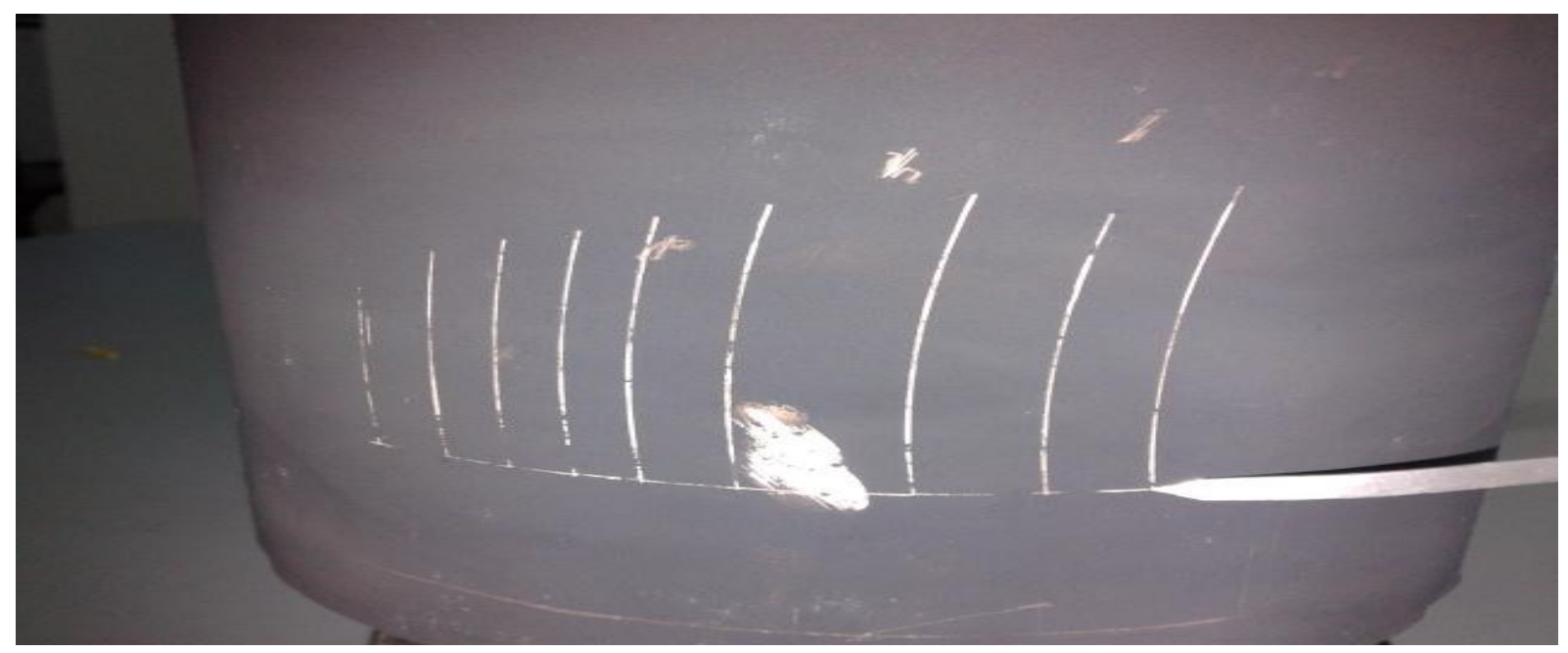

Fig. 11. Conventional kymograph result

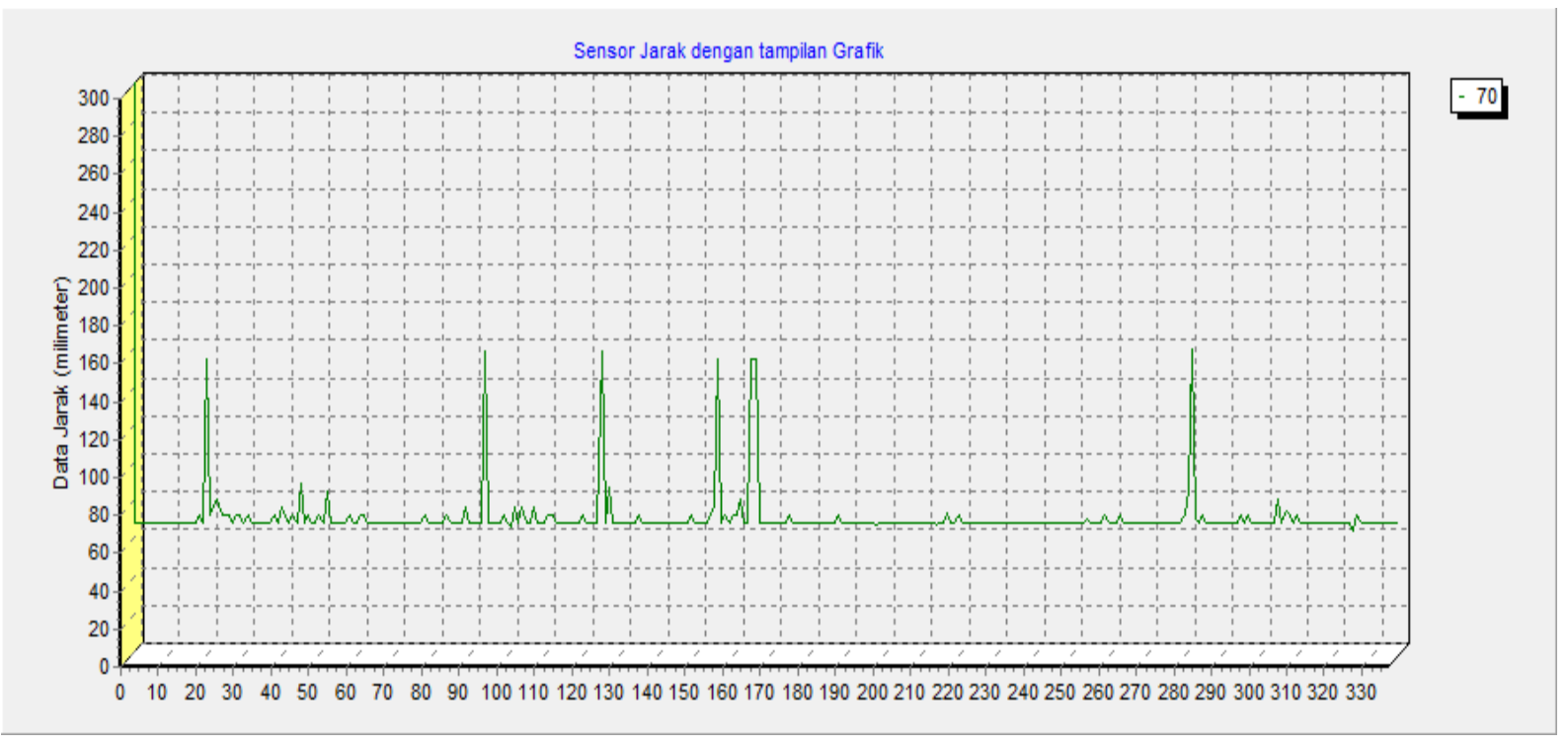

Fig. 12. . Computerized kymograph result

\section{RESULTS AND DISCUSSION}

Figure 12 shows the result of Computerized Kymograph, as our proposed device. From figure 11 and figure 12 above, we can compare the results of conventional kymograph (figure 11) and computerized kymograph (figure 12). It can be seen that our proposed device displays time value ( $\mathrm{x}$ axis) and distance value (y axis); while the conventional one do not provide those value but graphics.

Our device also provides more accurate result because it reduces friction force resulted from the conventional kymograph's drum. Force of friction can be calculated by [7]:

$$
F_{f}=\mu F_{N}
$$

Where:

$\mathrm{F}_{\mathrm{f}}$ is the force of friction in $\mathrm{N}$,

$\mu$ is the coefficient of friction, and

$\mathrm{F}_{\mathrm{N}}$ is the normal force in $\mathrm{N}$.

The value of $\mu$ depends on surface that dealing with.

By recording and displaying the result directly on the computer, we argue that the muscle contraction result on our device displays more accurate result than the conventional one. This also will lead better result's interpretation. Moreover, our device provides values on result, for example, the result (figure 12) shows that the maximum contraction 
achieved around $170 \mathrm{~mm}-180 \mathrm{~mm}$ at time (seconds) 20, 90, 120, 150, 162, and 278.

\section{CONCLUSION}

Our proposed device, computerized kymograph demonstrates better result by providing muscle contraction value. It is also easier to use than the conventional kymograph. Recently, the device has been used in our Medical Faculty, Yarsi University, Jakarta, Indonesia as a device to learn about physiology. The experiment on our laboratory shows that most of the students can interpret better using our device than the conventional one.

As an improvement in the future, we need to assess the device from user experience perspectives and device's functionality

\section{ACKNOWLEDGMENT}

We would like to express our gratitude to Indonesia Directorate General for Higher Education (DIKTI) for Research Grant awarded toward this research (Hibah unggulan DIKTI schema). We also would like to thank our institution (Yarsi University in Jakarta, Indonesia) for support and facility to test our device. We also appreciate for support and help from our colleagues in Department of Information Technology and Department of Medicine in Yarsi University.

REFERENCES

[1] A. Mukherjee, B. Jenkins, C. Fang, R.J. Radke, G. Banker, B. Roysam, Automated Kymograph Analysis for Profiling Axonal Transport of Secretory Granules,

[2] I. Smal, I. Grigoriev, A. Akhmanova, W. Niessen, E. Meijering, Microtubule dynamics analysis using kymographs and variable-rate particle filters, Image Processing, IEEE Transactions on 19, no. 7, pp. 1861-1876, 2010.

[3] O. Welzel, D. Boening, A. Stroebel, U. Reulbach, J. Klingauf, J. Kornhuber, T. Groemer, Determination of axonal transport velocities via image cross-and autocorrelation, European Biophysics Journal no. 38, pp. 883-889, 2009

[4] W. B. Ludington, W. F. Marshall, Automated analysis of intracellular motion using kymographs in 1, 2, and 3 dimensions, Vol. 7184, SPIE, 2009, URL http://link.aip.org/link/?PSI/7184/71840Y/1.

[5] http://www.atmel.com/images/doc2502.pdf accessed on 1 November 2013

[6] www.parallax.com/product/28015 accessed on 1 November 2013

[7] http://library.thinkquest.org/10796/ch4/ch4.htm accessed on 27 September 2013 